Ursula Baumann

Vom Recht auf den eigenen Tod

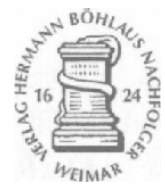




\section{Vom Recht \\ auf den eigenen Tod}

Die Geschichte des Suizids vom 18. bis zum 20. Jahrhundert

2001

Verlag Hermann Böhlaus Nachfolger Weimar 
Die Deutsche Bibliothek - CIP-Einheitsaufnahme

Baumann, Ursula:

Das Recht auf den eigenen Tod : die Geschichte des Suizids

vom 18. bis zum 20. Jahrhundert in Deutschland / Ursula Baumann.

- Weimar : Verl. Hermann Böhlaus Nachf., 2001

ISBN 978-3-7400-1180-2

ISBN 978-3-7400-1180-2

ISBN 978-3-476-02814-3 (cBook)

DOI 10.1007/978-3-476-(02814-3

Alle Rechte vorbehalten. Ohne schriftliche Genehmigung des Verlages ist es nicht gestattet, das Werk unter Verwendung mechanischer, elektronischer und anderer Systeme in irgendeiner Weise zu verarbeiten und zu verbreiten. Insbesondere vorbehalten sind die Rechte der Vervielfältigung - auch von Teilen des Werkes - auf photomechanischem oder ähnlichem Wege, der tontechnischen Wiedergabe, des Vortrages, der Funk- und Fernsehsendung, der Speicherung in Datenverarbeitungsanlagen, der Übersetzung und der literarischen oder anderweitigen Bearbeitung.

(C) 2001 Springer-Verlag GmbH Deutschland

Ursprünglich crschienen bei Verlag Hermann Böhlaus Nachfolger Weimar GmbH \& Co. 2001

www.boehlausnf.de

info@boehlausnf.de 


\section{Danksagung und Hinweise zur Notation}

\section{Danksagung}

Das vorliegende Buch ist die erweiterte Fassung meiner Habilitationsschrift, die im Sommersemester 2000 vom Fachbereich 1 der TU Berlin angenommen wurde. Ich danke der TU für das sechsmonatige Stipendium, das ich bis zur Beendigung meines Habilitationsverfahrens aus den Mitteln des Hochschulsonderprogamms zur Förderung des wissenschaftlichen Nachwuchses erhielt. Am Institut für Geschichtswissenschaft der TU war ich sechs Jahre lang als wissenschaftliche Assistentin tätig. Ohne die damit verbundene Absicherung und den mir hier zur Verfügung stehenden Forschungsfreiraum hätte ein so weitgespanntes und daher langwieriges Projekt nicht in Angriff genommen werden können. In Teilen der hiesigen scientific community war - ungeachtet aller neueren Entwicklungen in der Geschichtswissenschaft - das Verständnis für mein Arbeitsvorhaben nicht sehr groß. Vorurteile über das angeblich abseitige Thema begegneten mir in Colloquien immer wieder. Um so mehr muß ich die Bereitschaft anerkennen, mit der mich das Institut meinen Weg in völliger Selbständigkeit gehen ließ. Größten Dank schulde ich Reinhard Rürup, der den Mut hatte, mich mit diesem Projekt anzustellen. Den Ausbildungsauftrag der Universität schon immer außerordentlich ernst nehmend, bestand er zugunsten der Studierenden auf einem breiten Lehrangebot, was mir wiederum eine Vertiefung meiner geschichts- und gesellschaftstheoretischen Interessen ermöglichte, und gewährte mir ansonsten in der ihm eigenen Großzügigkeit Freiheit zur eigenständigen Forschung. Für ihre stete Hilfsbereitschaft bin ich Karin Hausen sehr dankbar, von ihrer konstruktiven Kritik haben nicht wenige Kapitel des Buches profitiert. Mein langjähriger "Zimmergenosse« am Institut, Rüdiger Hachtmann, schenkte mir seine überaus wohltuende kooperative Kollegialität. An der FU war es Christoph Conrad, an den ich mich mit sämtlichen professionellen Fragen wenden konnte. Vielfältige Unterstützung erhielt ich von Gisela Bock und Volker Hunecke, dem ich auch für seine engagierte Übernahme des Zweitgutachtens danke. Ute Frevert hat trotz außergewöhnlichen Belastungen noch den Part des auswärtigen Gutachtens übernommen, ich weiß, daß dies keineswegs selbstsverständlich ist.

Doris Kaufmann und François Guesnet standen mir all die Jahre in liebevollfreundschaftlicher Solidarität zur Seite, ihr kontinuierliches Nachfragen, ihre praktischen Hilfestellungen und die Weitergabe bibliographischer Funde haben die Arbeit sehr gefördert. In psychologischen und psychiatrischen Fragen der Suizidalität gewährten mir Rudolf Schmitt und Ingrid Wagener fachkundige Auskunft. Die Endphase der Habilitation bis zum Abschluß des Buchmanuskripts hat Claudia Sedlarz mit ebenso interessierter wie sorgfältiger Lektüre begleitet. 
Tilman Grammes hat mir durch seine kompetenten Ratschläge bei den Problemen, die mit der Wahl der Geistes- und Sozialwissenschaften als Beruf einhergehen, immer wieder das Durchhalten erleichtert. Von ihm, einem Pädagogen aus Leidenschaft, kamen viele nützliche Hinweise für meine Unterrichtstätigkeit. In dem oft mühsamen Prozeß der Klärung der Gedanken beim Schreiben hat mich von Anfang an Ursula Wolf auf alle nur erdenkliche Weise ermutigt. Sie unterzog sich als Philosophin ohne zu murren der Mühe, jedes Kapitel zu lesen, mit all dem Unrat, Staub und Geröll, den der Maulwurf der Geschichte ans Tageslicht fördert. Ihre präzisen Kommentare halfen mir, im Morast der Überlieferungen den Kopf oben zu behalten. Bei der oft komplizierten Beschaffung der Literatur hat Thorsten Wagner vorzügliche Arbeit geleistet, seine Intelligenz, Zuverlässigkeit und Freundlichkeit kamen dem Projekt sehr zugute. Auf den letzten Metern sprang für ihn schnell und unkompliziert Frederic Poetzsch ein. Bedanken möchte ich mich auch bei den Mitarbeiterinnen und Mitarbeitern der von mir besuchten Archive und Bibliotheken. Meine Eltern schenkten mir die ganze Zeit ihre unverminderte Anteilnahme, sie haben nicht nur regelmäßig meine Texte gelesen, sie nahmen auch klaglos hin, daß die älteste Tochter nach Abschluß des Habilitationsverfahrens plötzlich wieder von ihnen finanziell unterstützt werden mußte. Für die Publikation bewilligte die DFG einen Druckkostenzuschuß. Bernd Lutz vom Verlag Hermann Böhlaus Nachfolger Weimar bin ich für sein Engagement für das Buch sehr verbunden, seinen Mitarbeitern danke ich für die Arbeit der technischen Endredaktion.

\section{Hinweise zur Notation}

Auslassungszeichen in Zitaten verweisen immer auf Weglassungen von mir, die Markierung durch zusätzliche Klammern schien mir daher unnötig. Alle Hervorhebungen in Zitaten entstammen dem Original.

Um den Lesefluß zu erleichtern und die Argumente nicht zu stark hinter dem Zeitkolorit zurücktreten zu lassen, wurde die Orthographie der Quellen behutsam modernisiert.

Im Interesse der Leseökonomie habe ich auf die sprachliche Geschlechterparität verzichtet. Abgesehen von den Fällen, in denen es um konkrete einzelne Männer geht, werden die Termini "Suizident" und "Suizidenten" geschlechtsneutral verwendet. Zweifellos ist diese Praxis ein Tribut an eine fragwürdige Konvention; mangels einer überzeugenderen Lösung - die üblichen Alternativen scheinen mir für mein Unternehmen unangemessen - halte ich das jedoch für vertretbar. 


\section{Inhaltsverzeichnis}

Danksagung und Hinweise zur Notation $\ldots \ldots \ldots \ldots \ldots \ldots . \ldots$

Einleitung $\ldots \ldots \ldots \ldots \ldots \ldots \ldots \ldots \ldots \ldots \ldots \ldots \ldots \ldots \ldots \ldots \ldots \ldots \ldots \ldots \ldots \ldots$

I. Die lange Dauer der kirchlichen Macht -

Die Frage der Totenbestattung $\ldots \ldots \ldots \ldots \ldots \ldots \ldots \ldots \ldots$

II. Die Neuentdeckung eines alten Themas: zur "Aufklärung" des Suizids ................... 43

1. Im Handgemenge mit dem Zeitgeist: Theologen in der Defensive. . 47

2. Psychologische Erkundungen $\ldots \ldots \ldots \ldots \ldots \ldots \ldots \ldots \ldots \ldots$

3. Staatliche Interventionen und medizinische Deutungen $\ldots \ldots \ldots 83$

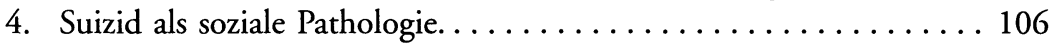

5. Radikale Aufklärung: Das Recht auf den »freiwilligen Tod « . . . . 127

\section{Gegenläufige Bewegungen im 19. Jahrhundert:}

Diskriminierung und Liberalisierung. . . . . . . . . . . . 145

1. Perspektiven der Hinterbliebenen . . . . . . . . . . . . . . 146

1.1 Eine Momentaufnahme aus dem Jahr 1811:

Die Grenzen öffentlicher Stellungnahme.... . . . . . 146

1.2 Momentaufnahmen 1812, 1817 und 1843:

Fürsorge und Mitgefühl. ................ 149

2. Rechtsgeschichte als Argument:

Der Abschluß der Entkriminalisierung des Suizids . . . . . . . . . 157

3. Leichenfleddereien. Die Institution der Anatomie und der Konflikt zwischen Wissenschaft und Pietät. . . . . . . . . 161

4. Jenseits von Gut und Böse: Zur Entmoralisierung des Suizids . . . . 180

5. Suizidzahlen und die Anfänge der Sozialwissenschaften . . . . . . . 202

5.1 Die Erfassung der Suizide . . . . . . . . . . . . . . . 205

5.2 Der Ansatz der Moralstatistik . . . . . . . . . . . . . . . 220

IV. Krisenstimmungen (1880-1914) ............... 227

1. Durkheim und seine Zeitgenossen: Der Suizid als fait social und der Entwurf einer Gesellschaftstheorie . . . . . . . . . . . 229

2. Daten und Interpretationen . . . . . . . . . . . 250 
3. Zwischen Heroisierung und Verachtung -

Einstellungen zur Selbsttötung und die Frage nach dem Wert

des Lebens

V. Zwischen Beihilfe zum Suizid und Tötung ohne Einwilligung: zur Diskussion über Sterbehilfe und Euthanasie im späten 19. und frühen 20. Jahrhundert .... 305

VI. Individuelles Unglück und kollektive Katastrophen. Vom Ersten Weltkrieg bis zum Ende des "Dritten Reichs" .......................... 323

1. Krieg und Republik. Die Permanenz der Krise ........... 323

2. Suizidalität im Nationalsozialismus und ihre Wahrnehmung in der Nachkriegszeit . . . . . . . . . . . . . . . . . . . . 348

Schlußbemerkung $\ldots \ldots \ldots \ldots \ldots \ldots \ldots \ldots \ldots \ldots \ldots \ldots \ldots \ldots \ldots$

Quellen- und Literaturverzeichnis.................. 389 